\title{
Preface to the viewpoint set on: Heterogeneous gradient and laminated materials
}

\author{
Lei $\mathrm{Lu}^{\mathrm{a}, *}$, Xiaolei $\mathrm{Wu}^{\mathrm{b}}$, Irene J. Beyerlein ${ }^{\mathrm{c}}$ \\ a Shenyang National Laboratory for Materials Science, Institute of Metal Research, Chinese Academy of Sciences, Shenyang 110016, China \\ ${ }^{\mathrm{b}}$ State Key Laboratory of Nonlinear Mechanics, Institute of Mechanics, Chinese Academy of Sciences, Beijing 100190, China \\ ${ }^{\mathrm{c}}$ Department of Mechanical Engineering, Materials Department, University of California, Santa Barbara, CA 93106-5070, United States
}

\section{A R T I C L E I N F O}

\section{Article history:}

Received 9 June 2020

Accepted 12 June 2020

Available online 24 June 2020

\section{Keywords:}

Heterogeneous

Gradient and laminated materials

\begin{abstract}
A B S T R A C T
The objective of the present Viewpoint set is to elucidate the state-of-art of this growing research field. Several key issues on mechanical properties/performance and mechanism of heterogeneous materials, mainly focus on gradient and laminated structures, will be addressed. Experimental studies combined with simulation and modeling are focusing on revealing the underlying mechanism of heterogeneous structures.
\end{abstract}

(c) 2020 Acta Materialia Inc. Published by Elsevier Ltd. All rights reserved.
The strength-ductility paradox has been a long-lasting issue for structural materials. Extensive studies over the past three decades indicate that homogenously refining coarse grains into ultrafine or even nanoscale can remarkably improve the strength/hardness and modestly improve fatigue life, but ductility is significantly diminished. In addition, the low thermal and mechanical stabilities of these nanostructured metals and alloys present limitations to their processing and applications. Therefore, developing strategies to improve the overall mechanical properties of nanostructured materials including strength, ductility, fatigue and fracture resistance as well as high stability has become vital to both materials science and their engineering applications.

Recently, materials with designed heterostructures have attracted growing attention of the materials community. There are a large number of natural and engineering heterostructures, which are typically characterized with appropriate geometry, distribution and multi-scale sizes spanning from micrometers even down to nanometers, including gradient, laminated, sandwiched, dual/multi- scale (or phases), and harmonic architecture. These emerging heterostructured materials generally exhibit unprecedented mechanical properties, such as, superior strength and ductility [1], extraordinary strain hardening [2,3], enhanced fatigue and fracture resistance [4] and remarkable resistance to wear, which are not accessible to their counterparts with homogenous or random mixed microstructures. The unique properties of heterostructured materials originate from the synergistic effect among the heterogeneities, which is resulted from the stress/strain gradient, geome-

\footnotetext{
* Corresponding author.

E-mail address: llu@imr.ac.cn (L. Lu).
}

try necessary dislocations, the interaction of new dislocation structures and unique interfacial behavior. To date, heterostructured materials have opened a new avenue towards understanding the heterostructure-related mechanical behaviors.

The objective of the present Viewpoint set is to elucidate the state-of-art of two major heterostructures: gradient structure and laminated structure, which refer to the gradient nanograined structure with built-in grain sizes from nanoscale in surface layer to coarse grains in the core and the 2-dimentional layered bimetal composites with individual layer thickness from micrometer to nanometer, respectively. Several key issues are addressed on mechanical properties/performance and mechanism of heterostructured materials, including strength-ductility, work hardening, fatigue and fracture behaviors, friction behavior, plastic deformation mechanism and stabilities. Focus is also placed on experimental studies combined with simulation and modeling to reveal the underlying mechanism of heterostructures.

This viewpoint set consists of seven invited articles. The first paper, contributed by $\mathrm{Wu}$, Zhu and Lu, deals with the mechanisms of strain hardening and plastic deformation in gradient- and lamellar structured materials, in which mechanical incompatibility leads to strain gradient and high strain hardening to produce a superior combination of strength and ductility. New strategies in heterostructured design based on hetero-deformation induced hardening, high density of hetero-interfaces, super-nanograins are discussed. Paper 2 by Liu and coauthors offers an alternative approach to enhance ductility in Fe-based alloy by engineering nano-scale heterogeneities. Through alloying with $\mathrm{Cu}$ and $\mathrm{Mn}$, a strong and very ductile advanced nanostructured Fe-based alloy was obtained, which is ascribed to the complex interplay between transforma- 
tion induced plasticity and the coherent nano-scale $\mathrm{Cu}$ precipitates as well as the heterogeneous stress-strain partitioning.

The next two articles focus on the fatigue and friction resistance of gradient nanograined metals. Lu et al. reviewed the basic feature of fatigue properties and mechanisms and found both lowcycle and high-cycle fatigue resistance can be greatly enhanced by introducing a gradient nanostructured skin on coarse-grained core. The all-round superior resistance are attributed to the delocalized cyclic deformation related with the spatially gradient distributed cyclic elastic and plastic strains and an ordered, progressive plastic yielding in the original built-in gradient grain size. Chen et al. summarized the novel strategy, along with the latest progress, for lowering the friction coefficients of metals via building gradient nano-grained surface layer (Scripta Materialia 185 (2020) 82-87). Several key aspects on deformation physics and friction behavior mechanisms are discussed, including surface morphology variation, subsurface structural evolution, and stress field.

Structural stability of nanostructures is vital for their practical applications both at elevated temperatures and under mechanical loading. $\mathrm{Lu}$ and $\mathrm{Li}$ overviewed the current progress in stabilization of nanograins with grain boundary relaxation, in which both thermal stability and mechanical stability of nanograins in several pure metals are enhanced significantly.

The last two papers give an overview of some theoretical issues. Gao and $\mathrm{Li}$ et al. combined experiments, theoretical and computational analysis concerning the relationship between the microstructure and mechanical properties in heterostructured metallic materials. Specifically, deformation behaviors and related models are highlighted from the mechanical view, e.g. strain gradient plasticity theory, which help us to understand the intrinsic structural heterogeneity and mechanical property improvements. Based on the effects of physical geometric nanoscale confinement,
Beyerlein et al. probed the defect-related processes occurring within the inter-layers of laminated metals. The influence of nano-confinement on strength, structure and radiation response of multilayers are presented in three different examples, also at interfaces in the nanoscale limit.

In summary, this collection of papers highlights many latest progresses, fundamental breakthroughs and future perspectives in the field of heterostructured gradient and laminated materials. Although advances in heterostructures have been achieved recently, there are still numerous critical issues related to designing, processing, characterization technique and fundamental understanding that need further exploration. Questions remain concerning the nature of heterostructures. The papers in this viewpoint set contribute to further our understanding of this fascinating new class of materials. Finally, the guest editors of this Viewpoint set would like to thank all the authors for their outstanding contributions.

\section{Declaration of Competing Interest}

The authors declare that they have no known competing financial interests or personal relationships that could have appeared to influence the work reported in this paper.

\section{References}

[1] T.H. Fang, W.L. Li, N.R. Tao, K. Lu, Revealing extraordinary intrinsic tensile plasticity in gradient nano-grained copper, Science 331 (2011) 1587-1590.

[2] Z. Cheng, H.F. Zhou, Q.H. Lu, H.J. Gao, L. Lu, Extra strengthening and work hardening in gradient nanotwinned metals, Science 362 (2018) 559-566.

[3] X.L. Wu, P. Jiang, L. Chen, F.P. Yuan, Y.T.T. Zhu, Extraordinary strain hardening by gradient structure, Proc. Natl. Acad. Sci. U S A, 111 (2014) 7197-7201.

[4] R.Q. Cao, Q. Yu, J. Pan, Y. Lin, A. Sweet, Y. Li, R.O. Ritchie, On the exceptional damage-tolerance of gradient metallic materials, Mater. Today 32 (2020) 94-107. 